\title{
Notes on Entropy for Concomitants of Record Values in Farlie-Gumbel-Morgenstern (FGM) Family
}

\author{
Saeid Tahmasebi \\ Persian Gulf University
}

\begin{abstract}
Let $\left\{\left(X_{i}, Y_{i}\right), i \geq 1\right\}$ be a sequence of bivariate random variables from a continuous distribution. If $\left\{R_{n}, n \geq 1\right\}$ is the sequence of record values in the sequence of $X$ 's, then the $Y$ which corresponds with the $n$ threcord will be called the concomitant of the $n$ th-record, denoted by $R_{[n]}$. In FGM family, we determine the amount of information contained in $R_{[n]}$ and compare it with amount of information given in $R_{n}$. Also, we show that the Kullback-Leibler distance among the concomitants of record values is distribution-free. Finally, we provide some numerical results of mutual information and Pearson correlation coefficient for measuring the amount of dependency between $R_{n}$ and $R_{[n]}$ in the copula model of FGM family.
\end{abstract}

Key words: Concomitants, Farlie-Gumbel-Morgenstern family, KullbackLeibler distance, mutual information, record values, Shannon entropy.

\section{Introduction}

Let $\left(X_{1}, Y_{1}\right),\left(X_{2}, Y_{2}\right), \cdots$ be a sequence of bivariate random variables from a continuous distribution. If $\left\{R_{n}, n \geq 1\right\}$ is the sequence of record values in the sequence of $X$ 's, then the $Y$ which corresponds with the $n$ th-record will be called the concomitant of the $n$ th-record, denoted by $R_{[n]}$. The concomitants of record values arise in a wide variety of practical experiments such as industrial stress testing, life time experiments, meteorological analysis, sporting matches and some other experimental fields. For other important applications of record values and their concomitants see Arnold et al. (1998) and Ahsanullah (1995). Some properties from concomitants of record values were discussed in Houchens (1984), Nevzorov and Ahsanullah (2000). The cumulative distribution function (cdf) for the FGM family is given by Johnson and Kotz (1975) as

$$
F_{X, Y}(x, y)=F_{X}(x) F_{Y}(y)\left[1+\alpha\left(1-F_{X}(x)\right)\left(1-F_{Y}(y)\right)\right], \quad-1 \leq \alpha \leq 1,
$$

where $F_{X}(x), F_{Y}(y)$ are marginal cdf of $X$ and $Y$, respectively. The copula model for this family is defined by Nelson (1999) as follows:

$$
f_{X, Y}(x, y)=[1+\alpha(-1+2 x)(-1+2 y)], \quad 0 \leq x, y \leq 1 .
$$


Houchens (1984) has obtained the probability density function (pdf) of concomitant of $n$ th-record value for $n \geq 1$ arising in (1) as

$$
h_{[n]}(y)=\int_{-\infty}^{+\infty} f(y \mid x) g_{n}(x) d x=f_{Y}(y)\left[1+\alpha_{n}\left(2 F_{Y}(y)-1\right)\right],
$$

where $\alpha_{n}=\alpha\left(1-2^{1-n}\right)$ and pdf of $R_{n}$ is

$$
g_{n}(x)=\frac{1}{(n-1) !}\left[-\ln \left(1-F_{X}(x)\right)\right]^{n-1} f_{X}(x) .
$$

Also, the joint density function of $R_{n}, R_{[n]}$ for the FGM copula is given by

$$
f_{n,[n]}(x, y)=[1+\alpha(1-2 x)(1-2 y)] \frac{[-\ln (1-x)]^{n-1}}{(n-1) !} .
$$

The information measures for record values have been investigated by several authors, including, Zahedi and Shakil (2006), Baratpour et al. (2007), and Madadi and Tata (2009). Amini and Ahmadi (2007) investigate the properties of Fisher information in the sequence of the first $\mathrm{n}$ records and their concomitants. Tahmasebi and Behboodian (2012) obtained some results of information measures for concomitants of order statistics. Recently, Fashandi and Ahmadi (2012) studied characterizations of symmetric distributions based on Rényi entropy of concocomiants. Shannon's entropy of a continuous random variable $X$, with pdf $f_{X}(x)$, is given by

$$
H(X)=-\int_{-\infty}^{+\infty} f_{X}(x) \ln f_{X}(x) d x .
$$

This is a mathematical measure of information which measures the average reduction of uncertainty of $X$. The organization of this article is as follows. In Section 2, we determine the amount of information contained in $R_{[n]}$ and compare it with amount of information given in $R_{n}$. In Section 3 , we show that the Kullback-Leibler distance between concomitants of $n$ th- and $m$ th- record values in FGM family is free from marginal distributions. Also, we present analytical expressions and some numerical results of mutual information and Pearson correlation coefficient between $R_{n}$ and $R_{[n]}$ in the copula model of FGM family.

\section{Entropy for Concomitants of Record Values in FGM Family}

Theorem 2.1. Let $\left(X_{i}, Y_{i}\right), i=1,2, \cdots$ be a sequence of independent observations from (1). If $R_{[n]}$ is the concomitant of the $n$ th-record value on the $X$ sequence of observations, then the Shannon entropy of $R_{[n]}$ for $n>1$, and $\alpha \neq 0$ is given by

$$
H\left(R_{[n]}\right)=C_{\alpha}(n)+H(Y)\left(1-\alpha_{n}\right)-2 \alpha_{n} \phi(f),
$$


where

$$
C_{\alpha}(n)=\frac{1}{8 \alpha_{n}}\left\{\left(1-\alpha_{n}\right)^{2}\left[2 \ln \left(1-\alpha_{n}\right)-1\right]-\left(1+\alpha_{n}\right)^{2}\left[2 \ln \left(1+\alpha_{n}\right)-1\right]\right\},
$$

and

$$
\phi(f)=\int_{0}^{1} u \ln f_{Y}\left(F_{Y}^{-1}(u)\right) d u .
$$

Proof. From (3) and (6), we get

$$
\begin{aligned}
H\left(R_{[n]}\right)= & -E_{h_{[n]}}\left[\ln f_{Y}(Y)\right]-E_{h_{[n]}}\left[\ln \left(1+\alpha_{n}\left(2 F_{Y}(Y)-1\right)\right)\right] \\
= & H(Y)\left(1-\alpha_{n}\right)-2 \alpha_{n} \int_{0}^{1} u \ln f_{Y}\left(F_{Y}^{-1}(u)\right) d u \\
& -E_{h_{[n]}}\left[\ln \left(1+\alpha_{n}\left(2 F_{Y}(Y)-1\right)\right)\right]
\end{aligned}
$$

Now, we need to find $E_{h_{[n]}}\left[\ln \left(1+\alpha_{n}\left(2 F_{Y}(Y)-1\right)\right)\right]$. First, we write

$$
\begin{aligned}
T(r) & =E_{h_{[n]}}\left[\left(1+\alpha_{n}\left(2 F_{Y}(Y)-1\right)\right)^{r}\right]=\int_{-\infty}^{+\infty} f_{Y}(y)\left[1+\alpha_{n}\left(2 F_{Y}(y)-1\right)\right]^{r+1} d y \\
& =\frac{1}{2 \alpha_{n}}\left[\frac{\left(1+\alpha_{n}\right)^{r+2}-\left(1-\alpha_{n}\right)^{r+2}}{r+2}\right] .
\end{aligned}
$$

Since the function under the integral sign in (10) is bounded by an integrable function. So, we have

$$
\begin{aligned}
& \left.\frac{-\partial T(r)}{\partial r}\right|_{r=0}=-E_{h_{[n]}}\left[\ln \left(1+\alpha_{n}\left(2 F_{Y}(Y)-1\right)\right)\right]=C_{\alpha}(n) \\
& =\frac{1}{8 \alpha_{n}}\left[\left(1-\alpha_{n}\right)^{2}\left(2 \ln \left(1-\alpha_{n}\right)-1\right)-\left(1+\alpha_{n}\right)^{2}\left(2 \ln \left(1+\alpha_{n}\right)-1\right)\right] .
\end{aligned}
$$

Putting (11) in (9) the result follows.

The difference between entropy of $(n+1)$ th- and $n$ th- concomitant of record values is obtained as

$$
\Delta(n)=H\left(R_{[n+1]}\right)-H\left(R_{[n]}\right)=C_{\alpha}(n+1)-C_{\alpha}(n)-\frac{\alpha}{2^{n}}[H(Y)+2 \phi(f)] .
$$

Note that $\lim _{n \rightarrow \infty} \Delta(n)=0$. A general expression for the entropy of the $n$ threcord value $R_{n}$ is presented by Zahedi and Shakil (2006) as

$$
\begin{aligned}
H\left(R_{n}\right)= & \ln (\Gamma(n))-(n-1) \psi(n) \\
& -\frac{1}{\Gamma(n)} \int_{-\infty}^{+\infty}\left[-\ln \left(1-F_{X}(x)\right)\right]^{n-1} f_{X}(x) \ln \left(f_{X}(x)\right) d x,
\end{aligned}
$$

where $\psi(n)$ is the digamma function. More recently, Baratpour et al. (2007) 
have explored the properties of $H\left(R_{n}\right)$. In the following examples we compare $H\left(R_{[n]}\right)$ with $H\left(R_{n}\right)$ in FGM family.

Example 2.1. Let $Z_{[n]}$ be concomitant of $n$ th-record value from (2), then, by using (3), the density function of $Z_{[n]}$ is

$$
p_{[n]}(u)=1+\alpha_{n}(2 u-1) .
$$

Now, by using (7) and (12), we can easily show that $H\left(Z_{[n]}\right)$ with $H\left(Z_{n}\right)$ for the copula model of FGM family have the following properties

(i) $H\left(Z_{[n]}\right)=C_{\alpha}(n)=C_{-\alpha}(n), \forall n>1$, and $\alpha \neq 0$.

(ii) $H\left(Z_{n}\right)=\log (\Gamma(n))-(n-1) \psi(n)$.

(iii) $H\left(Z_{n}\right) \leq H\left(Z_{[n]}\right)$.

(iv) $.5-\ln (2)<H\left(Z_{[n]}\right) \leq 0$, for $n \geq 1$, and $-1 \leq \alpha \leq 1$.

(v) for $n>1, H\left(Z_{[n]}\right)$ is increasing (decreasing) in $\alpha$ for $-1 \leq \alpha<0(0<\alpha \leq 1)$.

(vi) $H\left(Z_{[n]}\right)$ is decreasing in $n$ for $\alpha \neq 0$.

The relative differential entropy index, $\eta_{\alpha}(m, n)$, between $m$ th- and $n$ th- $(1<$ $m<n$ ) concomitant of record values, is given by

$$
\eta_{\alpha}(m, n)=\frac{H\left(Z_{[n]}\right)-H\left(Z_{[m]}\right)}{H\left(Z_{[n]}\right)-H\left(Z_{[1]}\right)}=\frac{C_{\alpha}(n)-C_{\alpha}(m)}{C_{\alpha}(n)} .
$$

Our numerical computations indicate when $n$ and $\alpha$ are fixed, then, $\eta_{\alpha}(m, n)$ for the copula model of FGM family is decreasing in $m$.

Remark 1. Using FGM example of Ebrahimi et al. (2010), insightful expression for $H\left(R_{[n]}\right)$ is given by

$$
H\left(R_{[n]}\right)=H\left(Z_{[n]}\right)-E_{h_{[n]}}\left[\ln f_{Y}(Y)\right],
$$

where $H\left(Z_{[n]}\right)=C_{\alpha}(n)$ is the entropy for $n$ th- concomitant of record value in the copula model of FGM family.

Remark 2. The following representation gives the Rényi entropy of order $\beta$ for $R_{[n]}$ in FGM family as

$$
R_{\beta}^{*}\left(R_{[n]}\right)=\frac{1}{1-\beta} \ln E_{U}\left\{\left[p_{[n]}(U)\right]^{\beta}\left[f_{Y}\left(F_{Y}^{-1}(U)\right)\right]^{\beta-1}\right\},
$$

where $U$ is a uniform random variable with parameters 0 and 1 . 
Example 2.2. Let $\left(X_{i}, Y_{i}\right), i=1,2, \cdots$ be a sequence of independent observations from Gumbel's bivariate exponential distribution with cdf

$$
\begin{aligned}
F(x, y) & =\left(1-\exp \left(\frac{-x}{\theta_{1}}\right)\right)\left(1-\exp \left(\frac{-y}{\theta_{2}}\right)\right)\left[1+\alpha \exp \left(\frac{-x}{\theta_{1}}-\frac{y}{\theta_{2}}\right)\right], \\
& -1 \leq \alpha \leq 1, \quad x, y>0, \quad \theta_{1}, \theta_{2}>0 .
\end{aligned}
$$

Then in this case by using (3), the pdf of $R_{[n]}$ is

$$
h_{[n]}(y)=\frac{1}{\theta_{2}} e^{\frac{-y}{\theta_{2}}}\left[1+\alpha_{n}\left(1-2 e^{\frac{-y}{\theta_{2}}}\right)\right] .
$$

Now, with using (7), we have

$$
H\left(R_{[n]}\right)=C_{\alpha}(n)+\left(1+\ln \left(\theta_{2}\right)\right)+\frac{\alpha_{n}}{2} .
$$

If we put $\theta_{1}=\theta_{2}$, then by using (12), (15) and numerical computations, we have $B_{\alpha}(n)=H\left(R_{[n]}\right)-H\left(R_{n}\right)=C_{\alpha}(n)+1+\frac{\alpha_{n}}{2}-\ln (\Gamma(n))+(n-1) \psi(n)-n>0$.

It is easy to check the following properties regarding $H\left(R_{[n]}\right)$ from FGM type Gumbel's bivariate exponential distribution.

(i) $H\left(R_{[n]}\right)$ is monotone increasing in $\alpha$, for $n>1 ; \forall \theta_{2}>0$.

(ii) $H\left(R_{[n]}\right)$ is an increasing concave function of $\theta_{2}$, for $n>1, \alpha \neq 0$.

(iii) $H\left(R_{[n]}\right)$ is increasing (decreasing) in $n$, for $0<\alpha \leq 1(-1 \leq \alpha<0)$.

Example 2.3. Suppose $\left(X_{i}, Y_{i}\right), i=1,2, \cdots$ be a sequence of independent observations from (1) with cdf

$$
F(x, y)=\left(1-x^{-\lambda_{1}}\right)\left(1-y^{-\lambda_{2}}\right)\left[1+\alpha\left(x^{-\lambda_{1}}\right)\left(y^{-\lambda_{2}}\right)\right], \quad x, y>1, \quad \lambda_{1}, \lambda_{2}>0 .
$$

In this case, by using (3), the pdf of $R_{[n]}$ is

$$
h_{[n]}(y)=\lambda_{2} y^{-\left(\lambda_{2}+1\right)}\left[1+\alpha_{n}\left(1-2 y^{-\lambda_{2}}\right)\right], \quad y>1 .
$$

By using (7), we get

$$
H\left(R_{[n]}\right)=C_{\alpha}(n)-\ln \lambda_{2}+\frac{\lambda_{2}+1}{\lambda_{2}}\left(1+\frac{\alpha_{n}}{2}\right) .
$$

Now, if we put $\lambda_{1}=\lambda_{2}=\lambda$, then by using (12), (16) and numerical computations, we have

$J_{\alpha}(n)=H\left(R_{[n]}\right)-H\left(R_{n}\right)=C_{\alpha}(n)-\ln \Gamma(n)+(n-1) \psi(n)+\frac{\lambda+1}{\lambda}\left(1+\frac{\alpha_{n}}{2}-n\right)<0$.

It is easy to check the following properties of $H\left(R_{[n]}\right)$ as: 
(i) $H\left(R_{[n]}\right)$ is monotone decreasing in $\lambda_{2}$, for $n>1, \alpha \neq 0$.

(ii) $H\left(R_{[n]}\right)$ is monotone increasing in $\alpha$, for $n>1 ; \forall \lambda_{2}>0$.

(iii) $H\left(R_{[n]}\right)$ is increasing (decreasing) in $n$, for $0<\alpha \leq 1(-1 \leq \alpha<0)$.

In the following theorem, we provide entropy bounds for concomitants of record values in FGM family.

Theorem 2.2. Let $R_{[n]}$ be the concomitant of the $n$ th-record value in FGM family. Then for $n>1$, and $\alpha \neq 0$, we have

$C_{\alpha}(n)+H(Y)\left[1-|\alpha|\left(1-2^{1-n}\right)\right] \leq H\left(R_{[n]}\right) \leq C_{\alpha}(n)+H(Y)\left[1+|\alpha|\left(1-2^{1-n}\right)\right]$.

Proof. From Theorem 2.1, we have

$$
H\left(R_{[n]}\right)=C_{\alpha}(n)+H(Y)+D_{\alpha}(n),
$$

where

$$
D_{\alpha}(n)=\alpha\left(1-2^{1-n}\right) \int_{-\infty}^{+\infty}\left(1-2 F_{Y}(y)\right) f_{Y}(y) \ln f_{Y}(y) d y,
$$

and $C_{\alpha}(n)$ is defined in (8). Since $-1 \leq 1-2 F_{Y}(y) \leq 1$, we have

$$
-|\alpha|\left(1-2^{1-n}\right) H(Y) \leq D_{\alpha}(n) \leq|\alpha|\left(1-2^{1-n}\right) H(Y) .
$$

Thus, by (18) and (19), the proof is clear.

\section{Kullback-Leibler Distance}

The Kullback-Leibler distance for two continuous random variables $Z_{1}$ and $Z_{2}$ with pdf's $f_{1}$ and $f_{2}$, respectively, is given by

$$
K\left(Z_{1}, Z_{2}\right)=\int_{-\infty}^{+\infty} f_{1}(z) \ln \left(\frac{f_{1}(z)}{f_{2}(z)}\right) d z=E_{1}\left(\ln \frac{f_{1}(Z)}{f_{2}(Z)}\right),
$$

where $E_{1}$ denotes the expectation with respect to $f_{1} . K\left(Z_{1}, Z_{2}\right) \geq 0$, and equality holds if and only if $f_{1}(z)=f_{2}(z)$ almost everywhere. It generalizes two measures of information, entropy and mutual information for communication theory.

Theorem 3.1. Let $R_{[n]}$ and $R_{[m]}$ be the concomitants of $n$ th- and $m$ th- record values in FGM family. Then the Kullback-Leibler distance between $R_{[n]}$ and $R_{[m]}$ is

$$
\begin{aligned}
K\left(R_{[n]}, R_{[m]}\right) & =K\left(Z_{[n]}, Z_{[m]}\right) \\
& =-C_{\alpha}(n)+\left(2^{-n}-2^{-m}\right) U_{\alpha}(m)+\frac{1-2^{1-n}}{1-2^{1-m}} C_{\alpha}(m),
\end{aligned}
$$


where

$$
\begin{aligned}
U_{\alpha}(m)= & \frac{1}{\alpha\left(1-2^{1-m}\right)^{2}}\left\{\left(1-\alpha_{m}\right)\left[\ln \left(1-\alpha_{m}\right)-1\right]\right. \\
& \left.-\left(1+\alpha_{m}\right)\left[\ln \left(1+\alpha_{m}\right)-1\right]\right\},
\end{aligned}
$$

and $C_{\alpha}(n)$ is defined in (8).

Proof. By using the invariance property of Kullback -Leibler information, the proof is easy.

Corollary 3.1. If $n=1$ and $m>1$, then, we obtain

$$
K\left(R_{[1]}, R_{[m]}\right)=-\int f_{Y}(y) \ln \left[1+\alpha_{m}\left(2 F_{Y}(y)-1\right)\right] d y=V_{\alpha}(m),
$$

where

$$
\left.V_{\alpha}(m)=\frac{1}{2 \alpha_{m}}\left\{\left(1-\alpha_{m}\right)\right)\left[\ln \left(1-\alpha_{m}\right)-1\right]-\left(1+\alpha_{m}\right)\left[\ln \left(1+\alpha_{m}\right)-1\right]\right\} .
$$

It is easy to see that $K\left(R_{[1]}, R_{[m]}\right)$ for $m \geq 1$, and $-1 \leq \alpha \leq 1$ has the following properties:

(i) $0 \leq K\left(R_{[1]}, R_{[m]}\right)<1-\ln 2$ for $m \geq 1$, and $-1 \leq \alpha \leq 1$.

(ii) for fixed $m>1, K\left(R_{[1]}, R_{[m]}\right)$ is decreasing (increasing) in $\alpha$ for $-1 \leq \alpha<0$ $(0<\alpha \leq 1)$.

(iii) $V_{\alpha}(m)$ is increasing in $m$ for $\alpha \neq 0$.

(iv) $V_{\alpha}(m)=V_{-\alpha}(m)$, for $m>1$, and $\alpha \neq 0$.

Corollary 3.2. If $m=1$ and $n>1$, then, we have

$$
K\left(R_{[n]}, R_{[1]}\right)=\int h_{[n]}(y) \ln \left(\frac{h_{[n]}(y)}{f_{Y}(y)}\right) d y=-C_{\alpha}(n),
$$

where $.5-\ln 2<C_{\alpha}(n) \leq 0$. Now, by using the results of Example 2.1 and Corollary 3.1, we can conclude that

$$
\min _{1 \leq n} K\left(R_{[n]}, R_{[1]}\right)=\min _{1 \leq m} K\left(R_{[1]}, R_{[m]}\right)=0 .
$$

For a bivariate random variable $(X, Y)$ with density function $f_{X, Y}(x, y)$ the mutual information is defined as

$$
I(X, Y)=H(Y)-H(Y \mid X),
$$


where $H(Y \mid X)$ is the conditional entropy of $Y$ given $X$. The mutual information is a generalization of the coefficient of determination, $\rho_{X Y}^{2}$, which unifies a variety of problems. We know that $I(X, Y) \geq 0$, and equality holds if and only if $X$ and $Y$ are statistically independent. Also, it has the invariance property under oneto-one transformation of $(X, Y)$.

Corollary 3.3. Let $R_{n}=F^{-1}\left(Z_{n}\right)$ be the $n$ th-record value and $R_{[n]}=F^{-1}\left(Z_{[n]}\right)$ be its concomitant obtained by a sequence from (1). Then, the mutual information between $R_{n}$ and $R_{[n]}$ for $n>1$ is distribution-free and is given by

$$
\begin{aligned}
I_{\alpha}\left(R_{n}, R_{[n]}\right) & =I_{\alpha}\left(Z_{n}, Z_{[n]}\right) \\
& =C_{\alpha}(n)+\int_{-1}^{1} \int_{-1}^{1} \ln (1+\alpha w v)(1+\alpha w v) \frac{\left[-\ln \left(\frac{1+w}{2}\right)\right]^{n-1}}{4(n-1) !} d v d w \\
& =C_{\alpha}(n)+E_{f_{n,[n]}}[\ln (1+\alpha W V)],
\end{aligned}
$$

where $W=1-2 X$ and $V=1-2 Y$.

Proof. By using the invariance property of mutual information, the proof is clear.

We can also obtain an explicit expression for the Pearson correlation coefficient between $R_{n}$ and $R_{[n]}$ in the copula model of FGM family. By using (5) and after some simple algebra, we get

$$
\rho_{\alpha}\left(R_{n}, R_{[n]}\right)=\frac{\alpha}{3} \sqrt{\frac{4^{n}-3^{n}}{12^{n-1}\left[1-\frac{\alpha^{2}}{3}\left(2^{1-n}-1\right)^{2}\right]}} .
$$

Table 1 provides the values of $I_{\alpha}\left(R_{n}, R_{[n]}\right)$ and $\rho_{\alpha}\left(R_{n}, R_{[n]}\right)$ as a function of $n$ and $\alpha$, for $n=1(1) 9$, and $\alpha=.2, .4, .8,1$. These values are derived by using (25), (26) and Maple software. Table 1 and easy computations show that $I_{\alpha}\left(R_{n}, R_{[n]}\right)$ and $\rho_{\alpha}\left(R_{n}, R_{[n]}\right)$ for the copula model of FGM family have the following properties:

(i) $I_{\alpha}\left(R_{n}, R_{[n]}\right)=I_{-\alpha}\left(R_{n}, R_{[n]}\right)$,

(ii) $\rho_{\alpha}\left(R_{n}, R_{[n]}\right)=-\rho_{-\alpha}\left(R_{n}, R_{[n]}\right)$,

(iii) $I_{\alpha}\left(R_{n}, R_{[n]}\right)<\left|\rho_{\alpha}\left(R_{n}, R_{[n]}\right)\right|$,

(iv) $I_{\alpha}\left(R_{n}, R_{[n]}\right)$ increases as $|\alpha|$ increases,

(v) $I_{\alpha}\left(R_{n}, R_{[n]}\right) \leq I_{\alpha}(X, Y) \leq 0.0599$,

(vi) $\left|\rho_{\alpha}\left(R_{n}, R_{[n]}\right)\right| \leq \alpha / 3$,

(vii) For fixed $\alpha \neq 0, I_{\alpha}\left(R_{n}, R_{[n]}\right)$ is decreasing in $n$. 
Table 1: $I_{\alpha}\left(R_{n}, R_{[n]}\right)$ and $\rho_{\alpha}\left(R_{n}, R_{[n]}\right)$ for the copula model of FGM family

\begin{tabular}{|c|c|c|c|c|c|c|c|c|}
\hline \multirow[b]{3}{*}{$n$} & \multicolumn{4}{|c|}{$I_{\alpha}\left(R_{n}, R_{[n]}\right)$} & \multicolumn{4}{|c|}{$\rho_{\alpha}\left(R_{n}, R_{[n]}\right)$} \\
\hline & \multicolumn{4}{|c|}{$\alpha$} & \multicolumn{4}{|c|}{$\alpha$} \\
\hline & 0.2 & 0.4 & 0.8 & 1 & 0.2 & 0.4 & 0.8 & 1 \\
\hline 1 & 0.0022 & 0.0089 & 0.0371 & 0.0599 & 0.0666 & 0.1333 & 0.2666 & 0.3333 \\
\hline 2 & 0.0013 & 0.0052 & 0.0227 & 0.0383 & 0.0510 & 0.1025 & 0.2093 & 0.2659 \\
\hline 3 & 0.0005 & 0.0023 & 0.0109 & 0.0202 & 0.0339 & 0.0686 & 0.1440 & 0.1874 \\
\hline 4 & 0.0002 & 0.0009 & 0.0047 & 0.0097 & 0.0213 & 0.0433 & 0.0927 & 0.1229 \\
\hline 5 & 0.00008 & 0.0003 & 0.0018 & 0.0043 & 0.0130 & 0.0265 & 0.0574 & 0.0769 \\
\hline 6 & 0.00003 & 0.0001 & 0.0007 & 0.0018 & 0.0078 & 0.0159 & 0.0346 & 0.0467 \\
\hline 7 & 0.000010 & 0.00004 & 0.0002 & 0.0007 & 0.0046 & 0.0094 & 0.0206 & 0.0279 \\
\hline 8 & 0.000003 & 0.000015 & 0.000092 & 0.0003 & 0.0027 & 0.0055 & 0.0121 & 0.0164 \\
\hline 9 & 0.000001 & 0.000005 & 0.00003 & 0.0001 & 0.0015 & 0.0032 & 0.0071 & 0.0096 \\
\hline
\end{tabular}

\section{Acknowledgements}

I am grateful to the editor and referees for their helpful comments and suggestions which improved the presentation of the paper.

\section{References}

Ahsanullah, M. (1995). Record Statistics. Nova Science Publishers, New York.

Amini, M. and Ahmadi, J. (2007). Fisher information in record values and their concomitants about dependence and correlation parameters. Statistics and Probability Letters 77, 964-972.

Arnold, B. C., Balakrishnan, N. and Nagaraja, H. N. (1998). Records. Wiley, New York.

Baratpour, S., Ahmadi, J. and Arghami, N. R. (2007). Entropy properties of record statistics. Statistical Papers 48, 197-213.

Ebrahimi, N., Hamedani, G. G., Soofi, E. S. and Volkmer, H. (2010). A class of models for uncorrelated random variables. Journal of Multivariate Analysis 8, 1859-1871.

Fashandi, M. and Ahmadi, J. (2012). Characterizations of symmetric distributions based Rényi entropy. Statistics and Probability Letters 82, 798-804.

Houchens, R. L. (1984). Record Value Theory and Inference. Ph.D. Dissertation, University of California, Riverside, California. 
Johnson, N. L. and Kotz, S. (1975). On some generalized Farlie-GumbelMorgenstern distributions. Communication in Statistics - Theory and Methods 4, 415-427.

Madadi, M. and Tata, M. (2011). Shannon information in record data. Metrika $\mathbf{7 4}, 11-31$.

Nelson, R. B. (1999). An Introduction to Copulas. Lecture Notes in Statistics 139, Springer, New York.

Nevzorov, V. B. and Ahsanullah, M. (2000). Some distributions of induced records. Biometrical Journal 42, 1069-1081.

Tahmasebi, S. and Behboodian, J. (2012). Information properties for concomitants of order statistics in Farlie-Gumbel-Morgenstern (FGM) family. Communication in Statistics - Theory and Methods 41, 1954-1968.

Zahedi, H. and Shakil, M. (2006). Properties of entropies of record values in reliability and life testing context. Communication in Statistics - Theory and Methods 35, 997-1010.

Received April 26, 2012; accepted July 23, 2012.

Saeid Tahmasebi

Department of Statistics

Persian Gulf University

Boushehr 75168, Iran

tahmasebi@pgu.ac.ir 